\title{
Solid State Characterization of Dried Liposomes in The Presence of Sucrose and HPMC as Dispersing Matrix
}

\author{
Nur Aini Mulyadi, Helmy Yusuf and Noorma Rosita
}

Department of Pharmaceutics, Faculty of Pharmacy, University of Airlangga, Indonesia

(Received: August 17, 2017; Accepted: November 07, 2017; Published: January 30, 2018)

\begin{abstract}
Disaccharides, such as sucrose, have been reported to protect phospholipid membranes during drying, while HPMC is used as gel matrix to inhibit recrystallization. We have investigated the solid state characterization of dried liposome formulation (air-dried and freeze-dried) which was preserved in the presence of sucrose and dispersed in HPMC matrix. The dried liposomal featured cationic dimethyldioctadecylammonium (DDA) was produced to overcome detrimental phase separation in phospholipid membranes during manufacturing process. The effect of sucrose and HPMC on lipid phase behavior during dehydration were characterized including their thermodynamics by DSC, crystallinity by XRD, and liposome structure by SEM. Data revealed that formula contain 5\% sucrose and 7.5\% HPMC showed a miscible mixture and relatively less crystalline-forming properties that potentially construct a stable dried liposome. The results may aid in the development of dried-DDA liposomal formulation by using combination of sucrose and HPMC.
\end{abstract}

Key words: Liposome, Sucrose, HPMC, Air drying, Freeze drying, Phase Separation.

\section{Introduction}

Liposome is termed as microscopic spherical vesicles with bilayer membranes which is formed when the phospholipids are exposed to water-rich environments, due to their amphipathic nature (Bozzuto and Molinari, 2015). Liposomes have been widely used in pharmaceutical application because of their advantageous features including liposomes are good carriers for both hydrophilic and lipophilic drugs, liposome can decrease toxicity by ensuring target organ specificity, that might reduce the effective dose of the drug (Kaur, 2011).

The main issue in the liposomal formulation for the pharmaceutical industry is the scaling up of the production processes. The conventional methods to produce liposomes have serious limitations, such as the material loss and the difficulty of scaling up high pressures devices Wagner and Vorauer-Uhl, 2011). In this study, an alternative method to overcome this problem has been utilized by air-drying and freezedrying to produce dried liposomes product, which need a subsequent controlled hydration step in order to obtain multilamellar liposomes, known as proliposomes strategy (Nekkanti et al., 2016; Ramteke et al., 2008).

Nevertheless, physical factors such as freezing and drying can also affect the integrity of bilayer membranes. The drying process involves the removal of water from phospholipid membranes usually results in irreversible structural and functional damage (Chiantia et al., 2015; Wolkers et al., 2004; Prestrelski et al., 1992).

Preservation of dried liposome has been achieved by the discovery that sugars can protect membranes during dehydration (air-drying or freezedrying). Sugar molecules directly interact with the polar headgroups of phospholipids membranes by forming hydrogen bonds (Pereira et al., 2004, Disalvo et al., 2004), they act to replace water molecules bound to lipid head groups and maintain head group spacing in the dry state similar to that of the hydrated lipid (Ohtake et al., 2005; Ingvarsson et al., 2011).

Correspondence to: Helmy Yusuf, Airlangga Street, Number of 4 - 6, Surabaya, 60115, Indonesia, E-mail: helmy-yusuf@ff.unair.ac.id, Cell: +62-812-1737-4255 
The effect of sucrose on the phase behavior of phospholipid membranes has been studied extensively, in view of the finding that sucrose can prevent phase separation in membranes during drying (Izutsu et al., 1998; Randolph, 1997). In addition, we used hydroxy-propylmethylcellulose (HPMC) as dispersing matrix to prevent crystallization that might happen during manufacturing, such as drying, and storage (Tejaa et al., 2013). It is interesting to combine mechanism of sucrose and HPMC on the phase behavior of lipid mixtures during drying.

This present study observed production of dried liposome products by freeze-drying and air-drying and emphasizes the influence of the mixture composition on the liposomes structure by using scanning electron microscopy (SEM), the liposomes thermodynamic properties by using differential scanning calorimetry (DSC), and the liposomes crystallinity properties by using x-ray diffractometry (XRD). The informations on such characteristics may offer future perspective into the development of potential formulation of dehydrated liposomes.

\section{Materials and Methods}

Materials: Materials used in the study includes soybean phosphatidylcholine (SPC) (Lipoid GmBh, Germany), dimethyl-dioctadecylammoniumbromide (DDA) (Sigma Aldrich, Singapore), cholesterol (Sigma-Aldrich, Singapore), hydroxypropylmethylcellulose (HPMC) (Shin-Etsu,Japan) and sucrose (Sigma-Aldrich, Singapura). Methanol (E. Merck) was used of analytical grade as a solvent to facilitate the mixing of liposomal ingredients.

Dried liposomes preparation: Liposomes were prepared by thin film hydration technique. Briefly, all weighed amount lipids in mol ratio of $1: 1: 0,1$ (DDA : SPC : Cholesterol) were dissolved in methanol. Solvent was evaporated under vacuum for an hour. The lipid film was hydrated with prewarmed $\left(50^{\circ} \mathrm{C}\right)$ ssucrose solution $(5 \% \mathrm{w} / \mathrm{v}$ or $10 \% \mathrm{w} / \mathrm{v})$ under consistent stirring for 10 minutes. The formed liposomes were sonicated using waterbath sonicator for 5 minutes, then dispersed into a pre-formed HPMC gel $(2.5 \% \mathrm{w} / \mathrm{v}$ or $7.5 \% \mathrm{w} / \mathrm{v})$.
The liposomal formulation was subsequently divided into freeze drying process at $-20^{\circ} \mathrm{C}$ for 48 hours (FS1-FS4) and air drying process at $40^{\circ} \mathrm{C}$ for 72 hours (FSO1-FSO4) to obtain solid products. The formulas could be seen in the Table 1 .

Table 1. Prepared formulation of dried Liposome.

\begin{tabular}{lccc}
\hline Formulation & $\begin{array}{c}\text { Drying } \\
\text { process }\end{array}$ & $\begin{array}{c}\text { Sucrose } \\
\text { concentration }\end{array}$ & $\begin{array}{c}\text { HPMC } \\
\text { concentration }\end{array}$ \\
\hline FS1 & & $5 \%$ & $2.5 \%$ \\
FS2 & Freeze & $10 \%$ & $2.5 \%$ \\
FS3 & drying & $5 \%$ & $7.5 \%$ \\
FS4 & & $10 \%$ & $7.5 \%$ \\
FSO1 & & $5 \%$ & $2.5 \%$ \\
FSO2 & Air & $10 \%$ & $2.5 \%$ \\
FSO3 & drying & $5 \%$ & $7.5 \%$ \\
FSO4 & & $10 \%$ & $7.5 \%$ \\
\hline
\end{tabular}

Differential scanning calorimetry (DSC) analysis: Thermal properties of liposome formulation formed were examined by the DSC (differential scanning calorimeter) instrument. Heating rate is 10 ${ }^{\circ} \mathrm{C}$ per minute at a temperature range of $30-250{ }^{\circ} \mathrm{C}$. The transition temperature of the sample is observed through a generated thermogram and measured as endothermic peak minimum of phase transition during the heating scan.

$X$-ray diffractometry (XRD) analysis: Crystallinity of the dry product were examined by the XRD (X-ray diffractometer) instrument. Samples were weighed and inserted into the holder and the surface was leveled. Furthermore, the holder is inserted into the instrument with the following conditions: Cu metal target, voltage of $40 \mathrm{kV}$, current of $30 \mathrm{~mA}$, slit width of $0.2 \mathrm{inch}$. Data is collected by scanning mode $0.2-0.5^{\circ}$ per minute with a scanning distance $2 \varnothing=5-40^{\circ}$

Scanning electron microscopy (SEM) analysis: The structure of the entrapped liposomes as well as the solid matrix were observed by the SEM (scanning electrone microscope) instrument. Portions of the dried product were scattered and glued onto $25 \mathrm{~mm}$ diameter plates, which were attached to SEM specimen mounts. The specimens were sputter-coated with a layer of gold approximately $5 \mathrm{~nm}$ in thickness for 180 seconds and specimens were examined on 
magnification 5000x (TM 3000 Table top Microscope, Japan).

\section{Results and Discussion}

A. DSC Study of Glassy State of Sucrose in The Presence of HPMC: In the present study, the percentage of sucrose concentration were $5 \%$ and $10 \%$. It was previously reported that sucrose at concentration of $10 \%$ was sufficient to preserve liposomes during dehydration (Yusuf et al. 2017). During drying, sucrose was expected to form the glassy state and dried liposomes were preserved in such a sugar glass matrix. To study the glassy state of sucrose, we determined glass transition state for samples by using differential scanning calorimeter.

Each sample was scanned with differential scanning calorimeter at $10^{\circ} \mathrm{C} / \mathrm{min}$ and the glass transition temperatures (Tg) of the FS1-FS4 were observed between $60^{\circ} \mathrm{C}$ and $80^{\circ} \mathrm{C}$, the midpoint temperature was $76.29^{\circ} \mathrm{C}$ (Fig. 1). On the other hand, the glass transition temperatures $(\mathrm{Tg})$ of the FSO1FSO4 were observed between $75^{\circ} \mathrm{C}$ and $85^{\circ} \mathrm{C}$, the midpoint temperature was $77.40^{\circ} \mathrm{C}$. (Fig. 2). The glass transition state from DSC thermogram of liposomal samples were essentially identical to that of the sucrose system, as we expected.

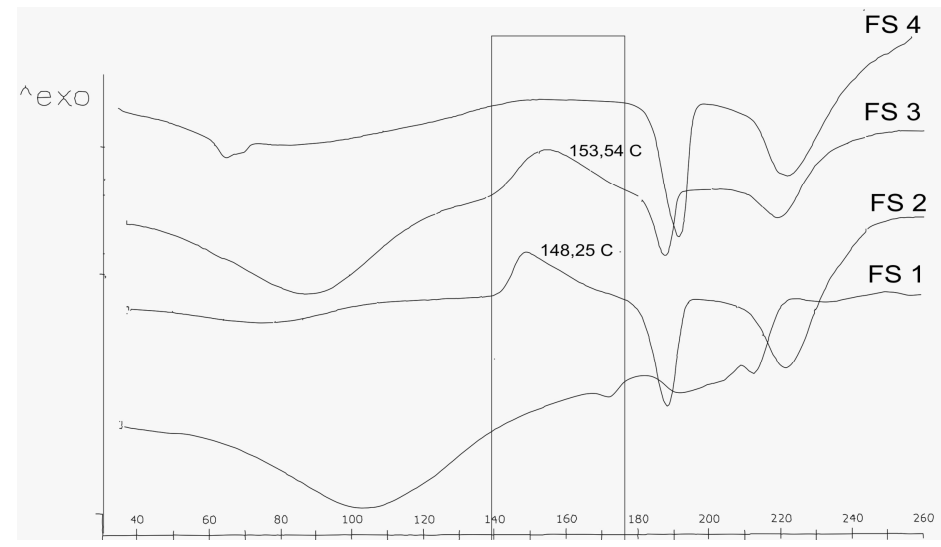

Figure 1. DSC thermogram of freeze-dried liposome formulation in the presence of sucrose and HPMC as dispersing matrix.

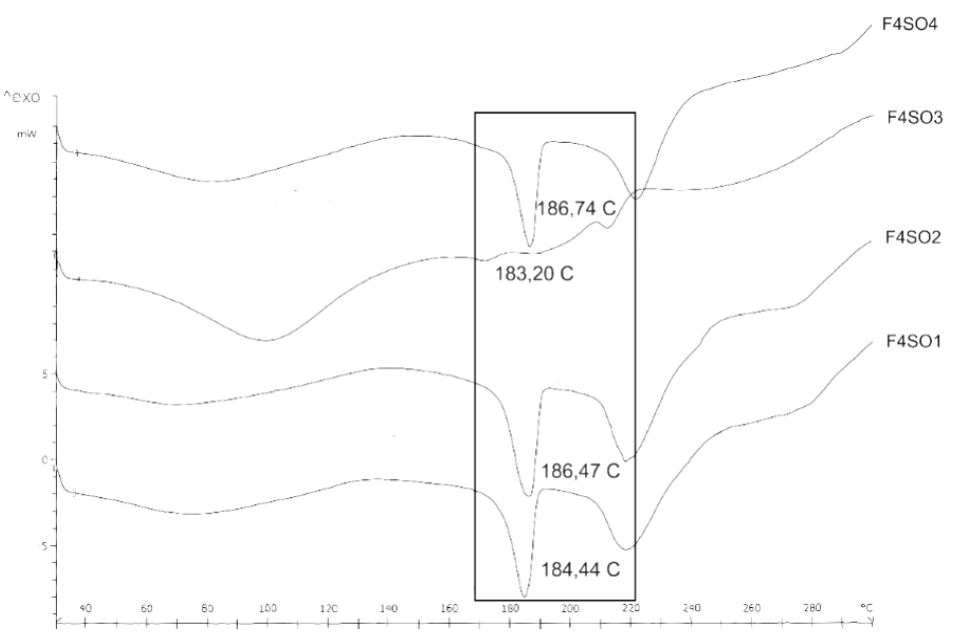

Figure 2. DSC thermogram ofair-dried liposome formulation in the presence of sucrose and HPMC as dispersing matrix 
Furthermore, only FS2 and FS3 as the freeze dried liposomes exhibited endothermic peaks respectively at $148.25^{\circ} \mathrm{C}$ and $153.54^{\circ} \mathrm{C}$, the midpoint temperature was $150^{\circ} \mathrm{C}$ (Fig. 1). On the other hand, all the air dried liposomes (FSO1-FSO4) exhibited endothermic peaks respectively at $184,44^{\circ} \mathrm{C} ; 186$, $47^{\circ} \mathrm{C} ; 183,20^{\circ} \mathrm{C}$; and $186,74^{\circ} \mathrm{C}$, the midpoint temperature was $185^{\circ} \mathrm{C}$ (Fig. 2). Thisendothermic event at $150^{\circ} \mathrm{C}$ and $185^{\circ} \mathrm{C}$ could be the melting temperature of sucrose crystal. The obtained solid product might be metastable as they underwent recrystallization upon heating. As already mentioned, several explanations have been offered in the literature for the appearance of melting peak for sucrose crystals is lower than that around $190^{\circ} \mathrm{C}$ (Bcckett et al. 2006). The possibility of the formation of a hydrate of sucrose and the inclusion of mother liquor in the crystal lattice or the presence of amorphous matter at the surface of the crystals has been mentioned (Lu et al. 2017). We concluded that this could be due to the devitrification of amorphous system which has been formed by combination of sucrose and HPMC matrix.

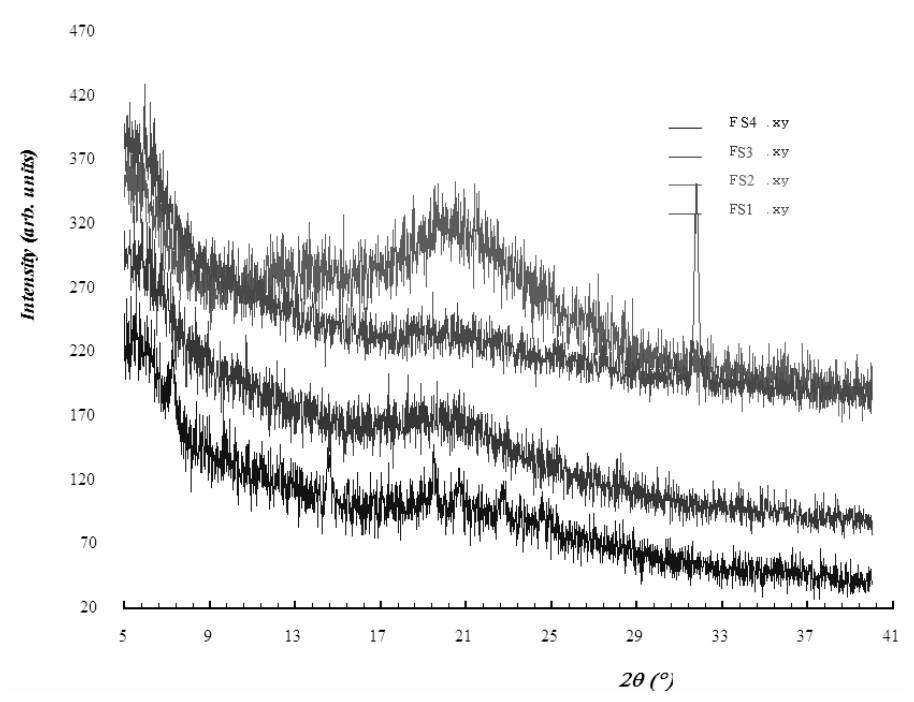

Figure 3. X-ray diffractograms of freeze-dried liposome formulation in the presence of sucrose and HPMC as dispersing matrix.

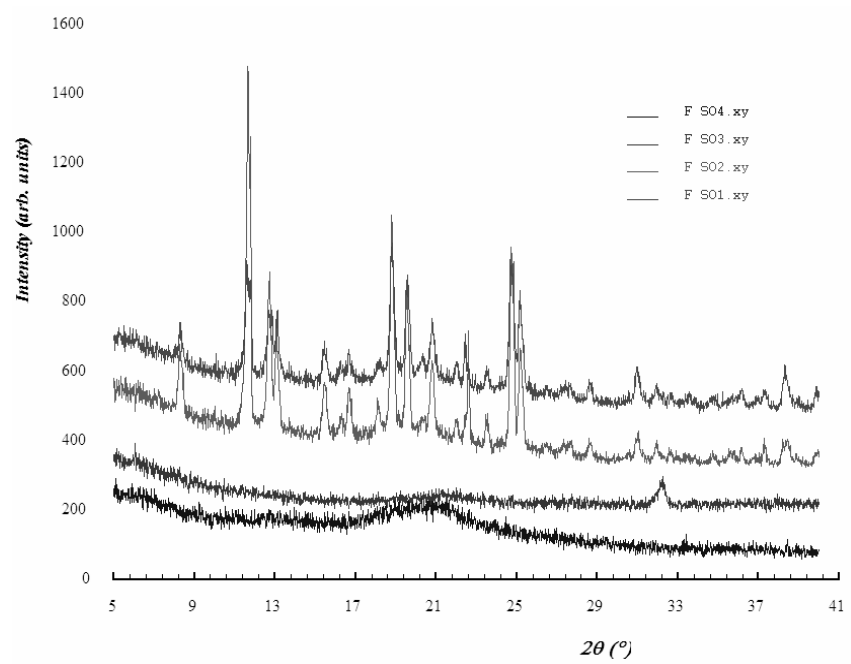

Figure 4. X-ray diffractograms of air-dried liposome formulation in the presence of sucrose and HPMC as dispersing matrix. 
B. XRD study of crystallinity of dried liposomes: In X-ray diffractograms, the crystallinity of samples is characterized by the shape (intensity or height and width) of the peaks. The narrower the peaks, the more crystalline the structures. The wide or small peaks indicate the presence of amorphous domains in the structures. Comparison of the resulting XRD diffractograms in Figs. 3-4 showed that the peaks differed in intensity.

The XRD diffractograms in fig. 3, FS1 and FS2 showed more intensive peaks and the same characteristics were observed in FSO1 and FSO2 in Fig. 4. On the other hand, FS3 and FS4 in fig. 3 showed much less intensive peaks and the same characteristics were observed in FSO3 and FSO4 in Fig. 4. It indicated that formula which contained HPMC in concentration of $2.5 \%$ still exhibited crystalline-forming properties, as we expected. The amorphous character of liposome system gives the benefits during hydration with water (Khadka et al. 2014)

Based on comparison of drying method, the air dried liposome has more crystalline-forming properties than the freeze dried liposomes. We concluded that it might happen because the heat energy from air drying was higher than from freeze drying, thus mixture of components are more enable to molecular re-arrangement in the system, as according to literature study that an amorphous material can crystallize when heat energy increased its molecular mobility (Yu et al., 2001).

C. SEM study of dried liposomes in the presence of HPMC matrix: The morphology of liposomes and the structure of solid matrices in figure 5 and 6 were imaged by SEM. The electronic micrographies showed that the liposomes were intactly observed in all formulations. FS4 and FSO4 which contained sucrose at concentration of $10 \%$ and also HPMC at concentration of $7.5 \%$ showed relatively larger vesicles which trapped in the solid matrices (fig. 6a and fig. 6b). In contrast, FS3 and FSO3 which contained sucrose at concentration of $5 \%$ and also with HPMC at concentration of $2.5 \%$ showed more solid matrices (Fig. 5a, b). Based on comparison of drying method, the freeze dried liposome was more porous than the air dried liposome, as according to literature study that a porous matrix is generally formed after freeze-drying ( $\mathrm{Yu}$ et al., 2001).

Furthermore, all liposomes were spherical shaped with smooth surface and intact in porous matrices structure. We concluded that such porous matrices are beneficial in terms of a more rapid reconstitution.

In this study, we have investigated the influence of combination of sucrose as a lyoprotectant agent and HPMC as a dispersing matrix agent on structural and physical characteristics of dried liposomal phase by air-drying and freeze-drying.

The preservation of liposome during drying was directly related to ability of sucrose to interact with the phospholipids. However, the physical separation of dried liposomes by bulky sucrose glass could be an important factor in preventing liposome in the glassy state (Simperler et al., 2006), because dynamic properties of the glassy state depend on high viscosity, which lowers the mobility of molecules (Wisitsorasak and Wolynes 2012), Ojovan 2008), and the physical separation of dried lipospmes could certainly inhibit the collapse membrane in the glass state (Ekdawj-Sever et al. 2001).

When compared to the hydrated system, dried mixtures display much higher phase transition temperatures. This is due to decreased head group spacing of the lipids in the dry state, which allows for more van der Waals interactions between lipid hydrocarbon chains, and thus higher onset of the chain melt. ${ }^{11}$ Nevertheless, the presence of disaccharides between the lipid head molecules, are known to act as water-structuring compounds, might be able to raise the attraction between the head groups of phosphoplipids, thus increase interfacial tension and as a result cause the lipid bilayers to become more closely packed (Ojovan 2008).

This is proven by the phase transitions occur at slightly higher temperatures in the presence of sucrose, at $76.29^{\circ} \mathrm{C}$ and $77.40^{\circ} \mathrm{C}$ (Figs 1-2). Thus, 
mixed phases of the lipid components are observed after drying. This is in sharp contrast with the situation before drying, in which the lipid components are phase separated at $40^{\circ} \mathrm{C}$ (the temperature of drying). We concluded that air-drying and freeze drying of this liposome mixture leads to mix of components and change to amorphous materials. Nevertheless, the thermodynamic instability of amorphous materials lead to a tendency

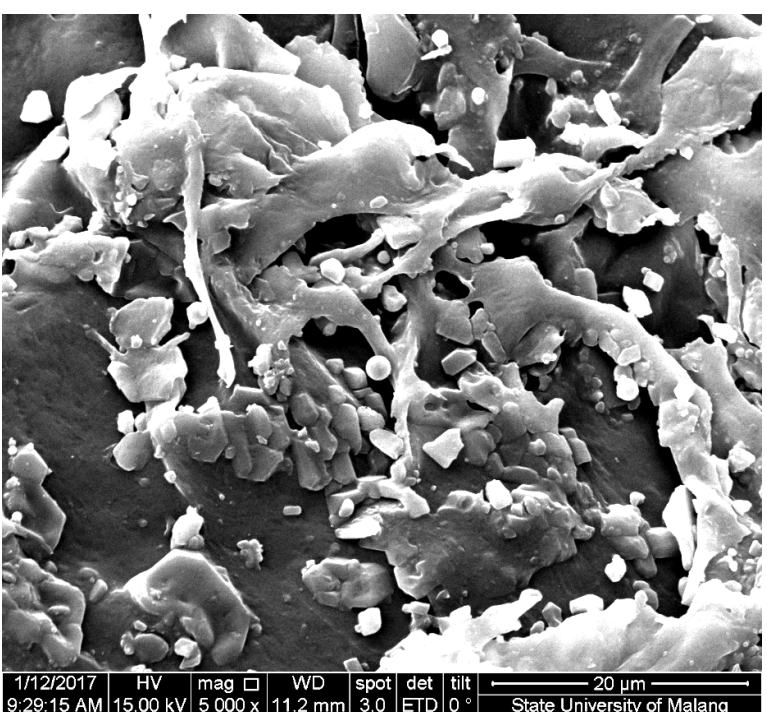

(a) FS3 to crystallize during storage. Crystallization occurs to a greater extent at temperatures above the glass transition temperature (Tg) (Leinen and Labuza 2006). The DSC spectra of freeze dried and air dried liposome system with sucrose showed that the recrystallization temperatures were $150{ }^{\circ} \mathrm{C}$ and $185^{\circ} \mathrm{C}$ respectively. These results indicated that dried liposomes, including amorphous systems, should be refrigerated to preserve their physical structures.

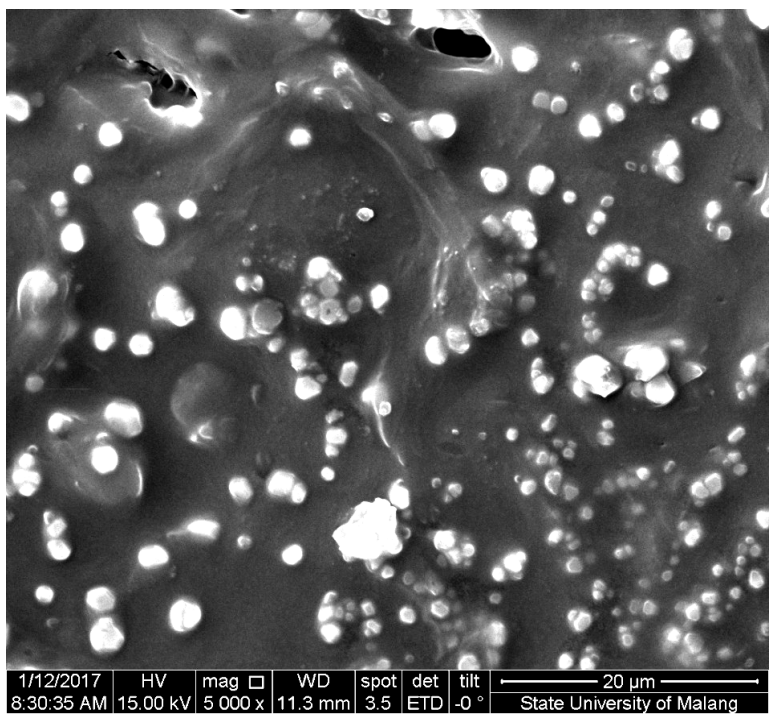

(b) $\mathrm{FSO} 3$

Figure 5. SEM image of dried liposome formulation in the presence of sucrose and HPMC as dispersing matrix : (a) FS3 and (b) FSO3.

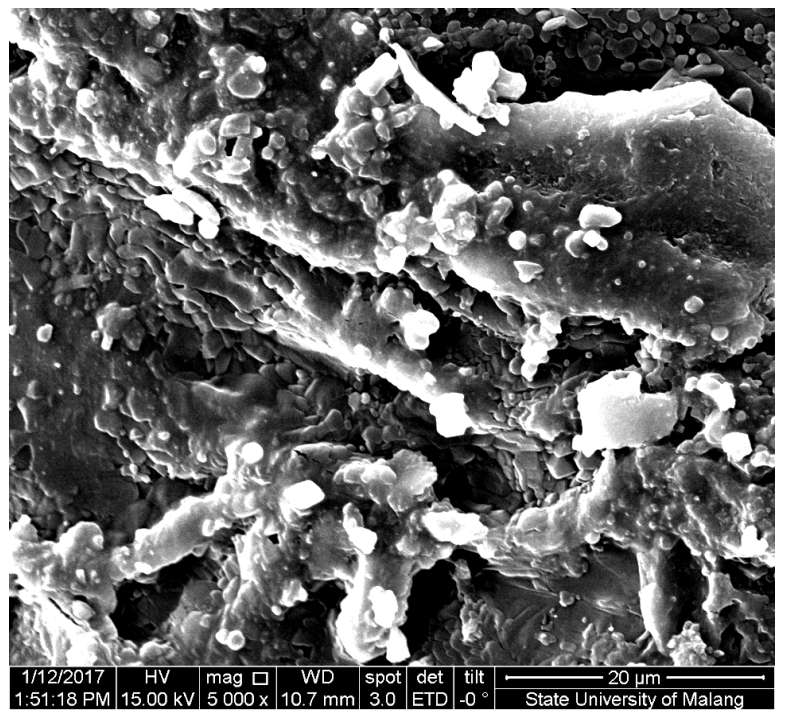

(a) FS4

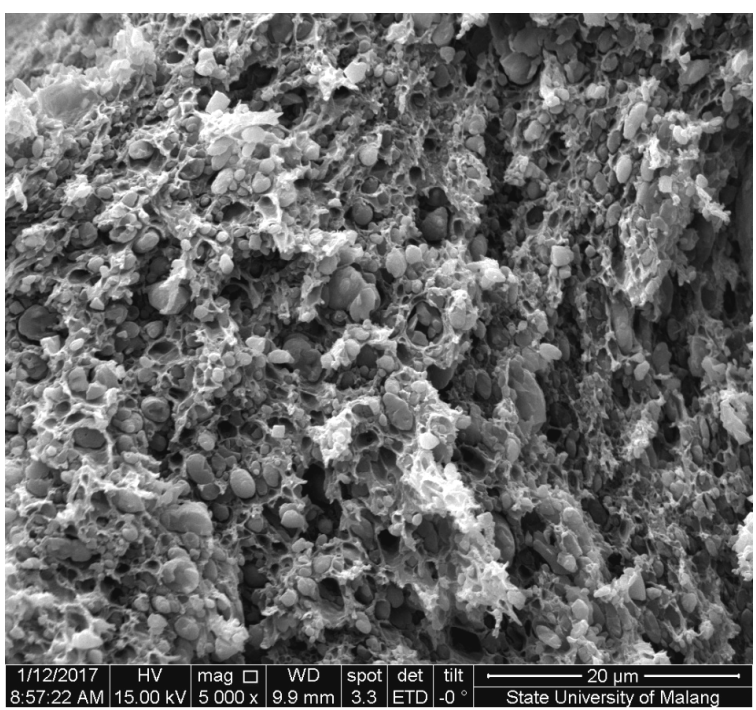

(b) $\mathrm{FSO} 4$

Figure 6. SEM image ofdried liposome formulation in the presence of sucrose and HPMC as dispersing matrix : (a) FS4 and (b) $\mathrm{FSO} 4$. 
Furthermore, formulations that contained sucrose at concentrations of $5 \% \mathrm{w} / \mathrm{v}$ with HPMC and at concentrations of $7.5 \% \mathrm{w} / \mathrm{v}$ did not lead to phase separation during drying, indicating a good miscibility of all components. The results showed that the HPMC matrix at concentration of $7.5 \% \mathrm{w} / \mathrm{v}$ might be sufficient in inhibiting recrystallization of all components upon manufacturing processes and storage. We concluded that formation of a new phase which does not undergo phase separation (miscible) could be a good sign that all components of liposome systems might lead to be a stable liposome with expected properties.

Nevertheless, sucrose at concentration of $10 \%$ w/v prefered to interact sucrose molecules with lipid molecules, thus the sharp peaks are strongly characteristic of the pure sucrose that nonincorporated in the resulting dried solid product. Moreover, HPMC matrix which was formed at concentration of $2.5 \%$ could not act as a adequate barrier to prevent recrystallization, thus phase separated happened from this mixture. In this case, we concluded that the combination of sucrose and HPMC as a function of concentration complied the purpose of preserving liposomal membrane.

\section{Conclusions}

The present study demonstrated the potential application of sucrose as lyoprotectant agent together with HPMC as dispersing matrix agent for the development of dried DDA-liposomal formulation. The amount of sucrose and HPMC in formulation significantly affect the phase behavior of lipid mixtures. The addition of sucrose at concentration of $5 \% \mathrm{w} / \mathrm{v}$ and HPMC at concentration of $7.5 \% \mathrm{w} / \mathrm{v}$ in dried liposomal formulation showed more miscible mixtures which preserved intact liposomes on amorphous and porous matrix. Controlling the phase transition and glass transition temperatures ensured preservation of the liposomal properties during manufacturing process and storage. The developed liposomal formulation in the form of dried solid products have wide range of pharmaceutical applications.

\section{Acknowledgements}

This research received funding form the Directorate General of Higher Education, The Republic of Indonesia.

\section{References}

Beckett, S.T., Francesconi, M.G., Geary, P.M., Mackenzie, G. and Maulny, A.P.E. 2006. DSC study of sucrose melting. Carbohydr. Res. 341, 2591-2599.

Bozzuto, G. and Molinari, A. 2015. Liposomes as nanomedical devices. Int. J. Nanomedicine, 10, 975999.

Chiantia, S., Kahya, N. and Schwille, P. 2005. Dehydration damage of domain-exhibiting supported bilayers: An AFM study on the protective effects of disaccharides and other stabilizing substances. Langmuir 21, 6317 6323.

Disalvo, E.A., Lairion, F., Martini, F., Tymczyszyn, E., Fras, M., Almaleck, H. and Gordillo, G.J. 2008. Structural and functional properties of hydration and confined water in membrane interfaces. Biochim. Biophys. Acta BBA - Biomembr. 1778, 2655-2670.

Ekdawi-Sever, N.C., Conrad, P.B. and de Pablo, J.J. 2001. Molecular simulation of sucrose solutions near the glass transition temperature. J. Phys. Chem. A. 105, 734-742.

Ingvarsson, P. T., Yang, M., Nielsen, H. M., Rantanen, J., and Foged, C. 2011. Stabilization of liposomes during drying. Expert Opin. Drug Deliv. 8, 375-388.

Izutsu, K., Heller, M.C., Randolph, T.W. and Carpenter, J.F. 1998. Effect of salts and sugars on phase separation of polyvinylpyrrolidone[ndash] dextran solutions induced by freeze-concentration. J. Chem. Soc. Faraday Trans .94, 411-417.

Kaur, R. 2011. Liposomes: Formulation and characterisation as contrast agents and as vaccine delivery systems, phd, Aston University. http://eprints.aston.ac.uk/15820/ (accessed March 13, 2016).

Khadka, P., Ro, J., Kim, Hyeongmin, Kim, I., Kim, J.T., Kim, Hyunil, Cho, J.M., Yun, G. and Lee, J. 2014. Pharmaceutical particle technologies: An approach to improve drug solubility, dissolution and bioavailability. Asian J. Pharm. Sci. 9, 304-316.

Leinen, K.M. and Labuza, T.P. 2006. Crystallization inhibition of an amorphous sucrose system using raffinose. J. Zhejiang Univ. Sci. B 7, 85-89.

Lu, Y., Yin, L., Gray, D.L., Thomas, L.C. and Schmidt, S.J. 2017. Impact of sucrose crystal composition and chemistry on its thermal behavior. J. Food Eng. 
Nekkanti, V., Venkatesan, N., Betageri, G. 2015. Proliposomes for oral delivery: Progress and challenges. Curr. Pharm. Biotechnol. 16, 303-331.

Ohtake, S., Schebor, C., Palecek, S.P. and de Pablo, J.J. 2005. Phase behavior of freeze-dried phospholipidcholesterol mixtures stabilized with trehalose. Biochim. Biophys. Acta BBA - Biomembr.1713, 57-64.

Ojovan, M.I. 2008. Viscosity and Glass Transition in Amorphous Oxides. Adv. Condens. Matter Phys. 2008, 1-23.

Ojovan, M.I. 2008. Viscosity and glass transition in amorphous oxides. Adv. Condens. Matter Phys. 1-23.

Pereira, C.S., Lins, R.D., Chandrasekhar, I., Freitas, L.C.G. and Hünenberger, P.H. 2004. Interaction of the disaccharide trehalose with a phospholipid bilayer: A Molecular dynamics study. Biophys. J. 86, 2273-2285.

Prestrelski, S.J., Tedeschi, N., Arakawa, T. and Carpenter, J.F. 1993. Dehydration-induced conformational transitions in proteins and their inhibition by stabilizers. Biophys. J. 65, 661-671.

Ramteke, S., Gupta, V. and Barupal, A. 2008. Formulation development and in vitro characterization of proliposomes for topical delivery of aceclofenac. Indian J. Pharm. Sci. 70, 768-775.

Randolph, T.W. 1997. Phase separation of excipients during lyophilization: Effects on protein stability. $J$. Pharm. Sci. 86, 1198-1203.

Simperler, A., Kornherr, A., Chopra, R., Bonnet, P.A., Jones, W., Motherwell, W.D.S. and Zifferer, G. 2006. Glass transition temperature of glucose, sucrose, and trehalose: An experimental and in silico study. $J$. Phys. Chem. B. 110, 19678-19684.
Tejaa, S.B., Patila, S.P., Shetea, G., Patelb,S. and Bansal, A.K. 2013. Drug-excipient behavior in polymeric amorphous solid dispersions. J. Excip. Food Chem. 4, 70-94.

Wagner, A. and Vorauer-Uhl, K. 2011. Liposome technology for industrial purposes. J. Drug Deliv. 1-9.

Wisitsorasak, A. and Wolynes, P.G. 2012. On the strength of glasses. Proc. Natl. Acad. Sci. 109, 16068-16072.

Wolkers, W.F., Oldenhof, H., Tablin, F. and Crowe, J.H. 2004. Preservation of dried liposomes in the presence of sugar and phosphate. Biochim. Biophys. Acta BBA Biomembr. 1661, 125-134.

Yu, L. 2001. Amorphous pharmaceutical solids: preparation, characterization and stabilization. $A d v$. Drug. Deliv. Rev. 48, 27-42.

Yusuf, H., Nugraheni, R., Mulyadi, N.A., Setyawan, D. and Rosita, N. 2017. Phase behavior of dried - DDA liposomal formulation dispersed in HPMC matrix in the presence of saccharides, Int. J. Pharm. Tech. Res. 10, 50-56.

Zhang, X., Guo, S., Gan, Y. and Yin, F. 2014. Preparation of redispersible liposomal dry powder using an ultrasonic spray freeze-drying technique for transdermal delivery of human epithelial growth factor. Int. J. Nanomedicine. 1665-1676 